\title{
Nanoparticles Against Viruses
}

Liudmila B. Boldyreva*

*Correspondence: boldyrev-m@yandex.ru

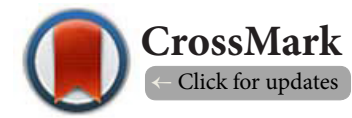

The State University of Management, Moscow, Russia.

\begin{abstract}
The main properties of viruses (they replicate inside the cell of all type of life forms, from animals and plants to microorganisms; have the shape from simple helical and icosahedral forms to more complex structures; are surrounded by protective protein "coat"; have very small size equal to the one-hundredth the size of most bacteria) determine the properties of the physical process that may influence viruses. It is shown in this work that spin supercurrent may be such a process.

One of the methods of influencing viruses is to change their form, for example, to deform and even unwind their helical forms. The spin supercurrent is distinct from other physical processes, most notably, in that it transforms angular momentum and, consequently, as a result of its action a change in the form of interacting objects may take place.

As spin supercurrent may transform angular momentum, then for a change in the form of viruses the spin supercurrent must emerge between viruses and objects whose form is similar to the form of viruses. Such objects may be 3D nanoparticles (NPs), for example, fullerenes and dendrimers.

The two aspects of influence of NPs on viruses are considered in this work: targeted drug delivery and change in viruses' form.
\end{abstract}

Keywords: Viruses, nanomedicine, metal nanoparticles, spin supercurrent, biophysics

\section{Introduction}

This article may be considered as a prolongation of author's article "The Physical Aspect of the Effects of Metal Nanoparticles on Biological Systems. Spin Supercurrents", published in the journal "Nanomaterials and Nanosciences" [1]. In the present article the peculiarities of acting of nanoparticles (NPs) on viruses are analyzed.

The main applications of metal NPs in medicine are: treatment; diagnosis; monitoring; control of diseases and targeted drug delivery (the most deep penetration into the tissue are performed by NPs of precious metals at passive targeting performed due to the properties of those NPs [2]).

By the main "physical" features of the effects of metal NPs on organs of a biological system are: the possibility of nonelectrostatic action [3], the non-monotonic size-effect dependence [4-5], the dependence on NP's form [4-6], the adhesion of certain metal NPs to specific organs' cells [7].

It is shown in [1] that the above-mentioned "physical" features of the effects of metal NPs on a biological system are determined by the properties of physical process accomplishing the interaction between metal NPs and the system; spin supercurrent is such a physical process.

The comparison of the properties of spin supercurrent and viruses [8] shows that the spin supercurrent may effectively influence viruses as well.

1) The viruses consist of one of the types of nucleic acid (DNA or RNA-Ribonucleic), that is, viruses are quantum objects having spin.

The spin supercurrent emerges between any objects having spin: electrically charged and neutral, magnetized and non-magnetized, constituting a living and non-living system.

2) The viruses are surrounded by a protective protein "coat".

The action of spin supercurrent is not shielded by electromagnetic and molecular screens.

3) The viruses have the shape from simple helical and icosahedral forms to more complex structures.

The spin supercurrent transforms angular momentum (in distinction from other physical processes) and, consequently, 
as a result of its action a change in the form of interacting objects may take place.

4) Viruses have very small size equal to the one-hundredth the size of most bacteria.

The effectivity of spin supercurrent's action is maximum if the interacting objects are in ultra-low doses.

Two aspects of influence of NPs on viruses are considered in this work: targeted drug delivery and influence on the form of viruses.

\section{Some properties of spin supercurrent}

The first attempt to describe the phenomenon of long transport of spin polarization (spin supercurrent) was made by $\mathrm{M}$. Vuorio [9]. In 2008, Russian scientists Y. Bunkov, V. Dmitriev, and I. Fomin were awarded the Fritz London Memorial Prize for their studies of spin supercurrent in superfluid ${ }^{3} \mathrm{He}-\mathrm{B}$ [10-12].

In superfluid ${ }^{3} \mathrm{He}-\mathrm{B}$, spin supercurrent emerges in the violation of the "stiffness" of spin part of the order parameter, that is under the non-zero difference in the values of respective angles of deflection and precession of precessing spins of ${ }^{3} \mathrm{He}$ atoms.

1) The value of spin supercurrent $\left(I_{S S}\right)_{z}$ in the direction of the orientation (axis $\boldsymbol{z}$ ) of the precession frequencies of the ${ }^{3} \mathrm{He}$ atoms' spins in superfluid ${ }^{3} \mathrm{He}-\mathrm{B}$ is determined to be

$$
\left(I_{S S}\right)_{z}=-g_{1} \partial \alpha / \partial z-g_{2} \partial \beta / \partial z,
$$

where $\alpha$ is the precession angle (phase), $\beta$ is the deflection angle, $g_{1}$ and $g_{2}$ are coefficients depending on $\beta$.

2) The action of spin supercurrent is aimed at equalizing the values of characteristics of spin structures between which it arises. Let us consider it in detail. The characteristics of interacting spin structures are given in Figure 1: $\mathbf{S}$ is spin, $\omega_{1}$ and $\omega_{2}$ are the spins' precession frequencies oriented along axis $\mathbf{z}, \alpha_{1}$ and $\alpha_{2}$ are the precession angles of spin's precession determined relative to reference line r.l., $\beta_{1}$ and $\beta_{2}$ are deflection angles.

Based on equation (1), spin supercurrent $\left(I_{S S}\right)_{z}$ between these spin structures may be written in the form:

$$
\left(I_{S S}\right)_{z}=-b_{1}\left(\alpha_{2}-\alpha_{1}\right)-b_{2}\left(\beta_{2}-\beta_{1}\right),
$$

where $b_{1}$ and $b_{2}$ are coefficients that are respectively depend on coefficients $g_{1}$ and $g_{2}$ introduced in equation (1); $b_{1}>0, b_{2}>0$. As a result of this action, the following inequalities hold:

$$
\begin{gathered}
\left|\beta_{2}^{\prime}-\beta_{1}^{\prime}\right|<\left|\beta_{2}-\beta_{1}\right| \\
\text { and } \\
\left|\alpha_{2}^{\prime}-\alpha_{1}^{\prime}\right|<\left|\alpha_{2}-\alpha_{1}\right|,
\end{gathered}
$$

where $\alpha_{1}$ and $\alpha_{2}$ are the values of precession angles $\alpha_{1}$ and $\alpha_{2}$ of spins of interacting spin structures after the action

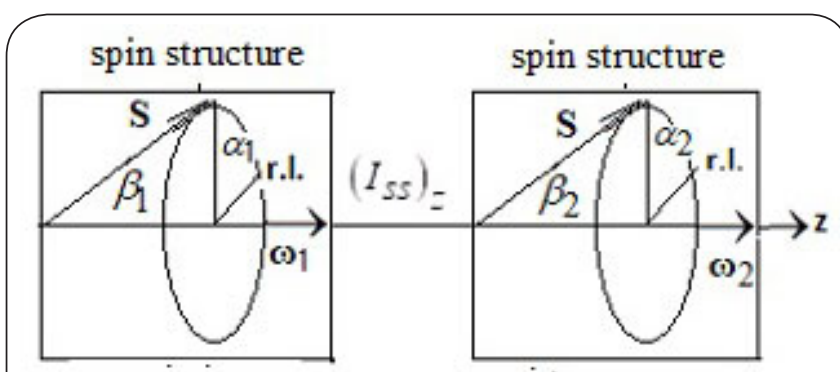

Figure 1. The schema of interaction of spin structures. $\left(I_{s s}\right)_{Z}$ is a spin supercurrent between spin structures; $\alpha_{1}$ and $\alpha_{2}$ are the precession angles; $\beta_{1}$ and $\beta_{2}$ are the deflection angles; $\omega_{1}$ and $\omega_{2}$ are the precession frequencies oriented along axis $\mathbf{z} ; \mathbf{S}$ is spin, r.l. is a reference line.

of spin supercurrent, $\beta_{1}^{\prime}$ and $\beta_{2}^{\prime}$ are the values of deflection angles $\beta_{1}$ and $\beta_{2}$ of spins of interacting spin structures after the action of spin supercurrent.

If to assume that before the action of spin supercurrent the precession angles $\alpha_{1}$ and $\alpha_{2}$ associated with the respective precession frequencies $\boldsymbol{\omega}_{1}$ and $\boldsymbol{\omega}_{2} \quad\left(\boldsymbol{\omega}_{1}\right.$ and $\boldsymbol{\omega}_{2}$ are taken to be independent of time $t$ ) as: $\alpha_{1}=\omega_{1} t$ and $\alpha_{2}=\omega_{2} t$, then from equation (4) it follows that one of the conditions of equalizing the precession angles is:

$$
\Delta \omega=\omega_{2}-\omega_{1} \rightarrow 0 \text {. }
$$

3) The spin supercurrent is not shielded by electromagnetic and molecular substances.

4) The action of spin supercurrents is most effective at small number of interacting spin structures. Let us estimate the total spin supercurrent $I_{\text {Sum }}$ emerging between an arbitrary spin structure and other $w$ spin structures. The total spin supercurrent

$$
I_{\text {sum }} \text { is determined to be } I_{\text {sum }}=\sum_{i=1}^{w} I_{i} \text {, where } I_{i} \text { is }
$$

the spin supercurrent between an arbitrary spin structure and the $i$-th spin structure from $w$ spin structures in question. Using equation (2), we obtain $I_{\text {sum }}=-\sum_{i=1}^{w}\left(b_{1} \Delta \alpha_{i}+b_{2} \Delta \beta_{i}\right)$, where $\Delta \alpha_{i}$ and $\Delta \beta_{i}$ are respectively the difference in the precession angles and the difference in deflection angles of spin structures determining current $I_{i}$. If all the values and signs of $\Delta \alpha_{i}$ and $\Delta \beta_{i}$ are respectively equiprobable and $w \rightarrow \infty$, then

$$
I_{\text {sum }} \rightarrow 0 \text {. }
$$

Condition (6) means that spin supercurrents cease to be the predominating factor that governs the result of interaction of considered spin structures and it will be determined by other physical factors. Thus, the action of spin supercurrents is most effective at a small number of interacting spin structures.

5) In 1949, R. Feynman for denotation of force fields in his diagrams [13] introduced virtual particles created by quan- 
tum objects. The properties of virtual particles depended on the interaction in which they were involved. For example, electric and magnetic interactions are accomplished by socalled virtual photons consisting of two oppositely charged virtual particles having spin. As virtual photon consists of two oppositely charged virtual particles it is characterized by electric dipole moment $\mathbf{d}_{v}$ and:

$$
\mathbf{d}_{v} \| \mathbf{S}_{v},
$$

where $\mathbf{S}_{v}$ is virtual photon's spin.

As, according to Feynman's model, every quantum object is a spin structure (as creating a virtual photon having spin), and spin supercurrent emerges between spin structures (the objects having spin), then spin supercurrent may emerge between any quantum objects.

\section{The adhesion of metal NPs to the cell surface of a biological system's organ}

Let us consider the properties of passive targeting of metal NPs.

1. The adhesion of metal NP to the cell surface of a biological system's organ is accomplished by non-electrostatic forces [3].

2. The adhesion of metal NP to the cell surface of a biological system's organ is the most effective in case where the substance that constitutes the NP has been contained in the organ before the action of the NP on the latter [7].

Both properties are due to features of spin supercurrent by means of which NPs "adhere" to the cell surface of a biological system's organ.

The first property is in accordance with the third characteristic of spin supercurrent, from which it follows that spin supercurrent is of non-electric and non-magnetic nature.

Let us analyze the second property in detail. According to conditions (3) and (4) spin supercurrent tends to equalize both the precession and deflection angles of the spins of virtual photons created by interacting quantum objects; as a result of action of this current the spins of these virtual photons ( $\mathbf{S}_{1}$ and $\mathbf{S}_{2}$, respectively) may be oriented in the same direction, that is

$$
\mathbf{S}_{1} \rightarrow \rightarrow \mathbf{S}_{2} \text {. }
$$

From conditions (7) and (8) it follows that two types of interaction may emerge between virtual photons and consequently between quantum objects creating these virtual photons: the first type - attractive electric dipole-dipole interaction [14]; the second type -attractive pseudomagnetic interaction having non-electromagnetic character.

Due to the fact that according to [3] non-electrostatic forces determine the adhesion of metal NPs to the cell surface, it follows that the pseudomagnetic force $(\mathbf{F})$ is predominant, see Figure 2.

\section{The pseudomagnetic interaction was discovered in the} following experiments.

- In the motion of nucleons in a substance with polarized spins of nuclei, a precession of spins of moving nucleons relative to the direction of substance's nuclei spin polarization takes place. The magnetic field does not affect this interaction, and the energy of the latter exceeds more than thousand times the energy of magnetic interaction [15] and [16].

- Ferromagnetism is caused by the formation of domains with ordered orientation of spins of electrons. The forces keeping those spins parallel are thousand times greater than the magnetic forces [17].

- In passing light through a magnetized medium, the light polarization twisting may take place. This phenomenon is called the Faraday effect and it is not a magnetic effect [18]. The Faraday effect demonstrates that not only spins of particles with nonzero rest mass but spins of photons may take part in pseudomagnetic interaction as well.

The accuracy of fulfilling the condition (8) which determines the existence of attractive force $\mathbf{F}$ (see Figure 2 ) depends on the accuracy of fulfilling the condition (5), that is on the difference between precession frequencies of virtual photons created by interacting quantum objects. Evidently, that in case of the adhesion of metal NP to the cell surface of a biological system's organ the condition (5) holds most accurately if the properties of NP are analogous to the properties of the organ. This explains the experimental fact that the metals whose NPs effectively "adhere" to certain organs are present in the organ before the introduction of NPs [4, 7]. For example, iron ions are known to be present in the form of reserve protein ferritin in the spleen; silver is contained in the brain, liver, kidneys, and bones; and gold is found in the blood.

\section{The influence of metal NPs on the viruses' form} According to conditions (3) and (4), spin supercurrent changes both the precession and deflection angles of the spins of virtual photons on which it acts, and thus changes the form

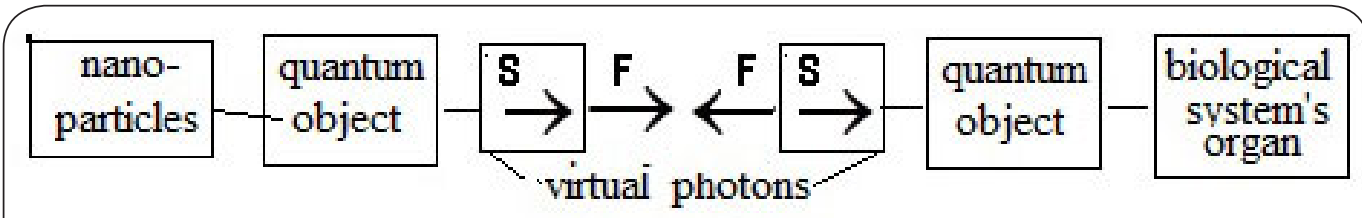

Figure 2. Attraction forces $\mathbf{F}$ between virtual particles created by quantum objects constituting nanoparticles (NPs), on the one hand, and biological system's organ, on the other hand. $\mathbf{S}$ are spins of virtual particles. 
of the quantum objects creating those virtual photons. It should be noted that the spin supercurrent differs from other physical processes, most notably, in that it transforms angular momentum and, consequently, as a result of its action a change in the form of interacting objects may take place.

Thus, spin supercurrent emerging between virtual photons created by quantum objects of NPs and virtual photons created by quantum objects of viruses may change the form of viruses, that is, change their physical properties. According to conditions (5) the effect of NP on BS is most pronounced, if the characteristics of NP and viruses are similar to each other, in particular, have similar forms.

This may account for the experimental fact that 3D NPs, which are spiral shaped, deform and even unwind the spiral when penetrating a DNA molecule. One example of such NPs are fullerenes (computer simulations have shown that fullerenes, namely, spherical C60 molecules, are potentially dangerous to DNA molecules [19].) Another example is dendrimers: 3D and higher generation dendrimers have a form which is similar to a sphere [20].

\section{The determination of the spin precession frequencies characterizing viruses}

In accordance with condition (5), for choosing the NP effectively influencing viruses it is necessary to know the spin precession frequencies of virtual photons created by quantum objects constituting NP and viruses. One of the methods of determining these frequencies is the use of values of energy spectrum of those quantum objects. The precession frequency $\omega_{v}$ of virtual photon is determined by energy $\boldsymbol{U}_{q}$ of quantum objects creating the virtual photon as [21]:

$$
\omega_{v}=U_{q} / \hbar \text {, }
$$

where $\hbar$ is Planck's constant.

However, it is very difficult to determine energy spectrum of quantum objects constituting viruses. In this case it is better to determine the precession frequencies characterizing viruses with the use of photons. According to [22] (Weber and Lynn 2000), frequency $\omega_{p h}$ of a circularly polarized photon is the frequency of precession of photon's spin. Being a quantum object, the photon may interact with quantum objects that constitute viruses by spin supercurrents. This interaction may be maximally effective at fulfilling the condition (5). Consequently, determining frequency $\omega_{p h}$ at which this interaction is maximally effective, using condition (5), may determine the spin precession frequency $\omega_{v i r}$, characterizing viruses, that is

$$
\omega_{\text {vir }} \approx \omega_{p h} \text {. }
$$

The determination of the photon frequency meeting condition (10) can be performed in the following way. The photon beam under study passes at a small distance from the biological system (for example, in experiments described in [23] the distance was $10-15 \mathrm{~mm}$ ). The amplitude and frequency modulation of the photon beam are measured. By varying the photon frequency, one may determine the frequency at which the impact on the photon beam is most pronounced for the given state of the biological system.

\section{Conclusion}

The 3D NPs, which are spiral shaped may be used for effective influencing viruses and this influence is accomplished by spin supercurrent possessing all necessary characteristics for effective influencing viruses:

- the spin supercurrent transforms angular momentum and, consequently, as a result of its action a change in the form of interacting objects may take place;

- the spin supercurrent may influence quantum objects constituting viruses in cells of all type of life forms;

- the spin supercurrent's influence is independent of the presence of cell's protective protein "coat";

- the spin supercurrent's influence is most pronounced if interacting objects are in ultra-low doses, the latter is one of main characteristics of viruses and NPs.

\section{Competing interests}

The author declares that he has no competing interests.

\section{Acknowledgements}

I am grateful to Mikhail A. Boldyrev for his assistance in translating this paper into English.

\section{Publication history}

EIC: Mallikarjuna Nadagouda, US Environmental Protection Agency, USA.

Received: 08-Jan-2021 Final Revised: 10-Mar-2021

Accepted: 25-Mar-2021 Published: 08-Apr-2021

\section{References}

1. L. B. Boldyreva. The Physical Aspect of the Effects of Metal NPs on Biological Systems. Spin Supercurrents. Nanomaterials and Nanosciences, 2(1), 7 pp., 2014.

2. G. Zhang, Z. Yang, W. Lu, et al., "Influence of anchoring ligands and particle size on the colloidal stability and in vivo biodistribution of polyethylene glycol-coated gold NPs in tumor-xenografted mice," Biomaterials, vol. 30(10), pp. 1928-1936, 2009.

3. G. Oberdorster, A. Maynard, K. Donaldson, et al., "Principles for characterizing the potential human health effects from exposure to nanomaterials: elements of a screening strategy. Particle and Fibre," Toxicology, vol. 2, no. 8, 2005.

4. E. M. Egorova, "Biological effects of silver NPs," In: Audrey E. Welles, editor. Silver NPs: Properties, Characterization and Applications. Chapter 5. Nova Science Publishers, Inc: 221-258, 2010.

5. C. N. Lok, C. M. Ho, R Chen, et al., "Silver NPs: partial oxidation and antibacterial activities," J. Biol. Inorg. Chem. vol. 12, pp. 527-534, 2007.

6. S. Pal, Y. K. Tak, a n d J. M. Song, "Does the antibacterial activity of silver NPs depend upon the shape of the NPs?" Applied and Environmental Microbiology, 73, no. 6, p. 1712, 2007.

7. T. Neuberger, B. Schöpf, H. Hofmann, M. Hofmann, and B. Rechenberg, "Superparamagnetic nanoparticles for biomedical applications: Possibilities and limitations of a new drug delivery system," Journal of Magnetism and Magnetic Materials, vol. 293, pp. 483-496, 2005.

8. M. J. Roossinck, "Virus”, Princeton: Princeton University Press. 2016.

9. M. Vuorio, "Relaxation by magnetic counterflow in superfluid ${ }^{3} \mathrm{He}^{\prime}$. J. 
Liudmila B. Boldyreva, Nanomaterials and Nanosciences 2021, http://www.hoajonline.com/journals/pdf/2053-0927-9-1.pdf

Phys. C: Solid State Phys., vol. 9(11), p. 267, 1976. DOI:10.1088/00223719/9/11/003.

10. A. S. Borovic-Romanov, Yu. M. Bunkov, V. V. Dmitriev, Yu. M. Mukharskii, and D. A. Sergatskov, "Investigation of Spin Supercurrents in ${ }^{3} \mathrm{He}-B$," Physical Review Letters, vol. 62, no.14, p. 1631, 1989.

11. Yu. M. Bunkov, "Spin Superfluidity and Coherent Spin Precession," Journal of Physics: Condensed Matter, vol. 21(16), pp. 164201-164206, 2009.

12. V. V. Dmitriev, and I. A. Fomin, "Homogeneously precessing domain in ${ }^{3} \mathrm{He}-\mathrm{B}$ : formation and properties," Journal of Physics: Condensed Matter, vol. 21(16), p. 164202, 2009.

13. R. Feynman, "Space-time approach to quantum electrodynamics". Phys. Rev. vol. 76, pp. 769-789, 1949.

14. E. Purcell, "Electricity and Magnetism. Berkeley physics course", vol. 2. New York: McGraw-Hill Book company, 1965.

15. A. Abragam and M. Goldman, "Nuclear magnetism: order \& disorder". Clarendon Press Oxford, 626 pp, 1982.

16. V. G. Pokazaniev and G. V. Skrotskii, "Pseudomagnetism". PhysicsUspekhi, vol. 129(4), pp 615-644, 1979 (in Russian).

17. A. Stephenson, "On the Curie points and Weiss molecular field coefficients of ferrimagnetic spinels." Phys. Let. A, vol. 43(Issue 3), pp. 295-296, 1973.

18. F. Richardson and J. Riehl, "Circularly polarized luminescence spectroscopy". Chem. Rev. vol. 77, p. 773, 1977.

19. X. Zhao, A. Striolo, and P. T. Cummings, "C60 Binds to and Deforms Nucleotides," Biophysical Journal, vol. 89, pp. 3856-3862, 2005.

20. Ba. K. Nanjwade, H. M. Bechra, G. K. Derkara, F. V. Manvia, and Ve. K. Nanjwade, "Dendrimers: Emerging polymers for drug-delivery systems," European Journal of Pharmaceutical Sciences (Elsevier), vol. 38(3), pp. 185-196, 2009.

21. L. B. Boldyreva, "Quantum Correlations-Spin Supercurrents". International Journal of Quantum Information, vol. 12(1), 1450007 (13 pp.), 2014

22. Weber M. and Lynn K. (2000). Three Photon Annihilations of Positrons and Positronium in Solids with Two Detectors in Coincidence. Radiat. Phys. Chem, 58(5-6):749-775.

23. Sargsyan, Rafik, Gagic Karamyan, and Mkrtich Avagyan. 2010. "Noninvasive Assessment of Physiologic State of Living Systems". Journal of Alternative \& Complementary Medicine 16(11): 1137-1144.

\section{Citation:}

Liudmila B. Boldyreva. Nanoparticles Against Viruses. Nanomater Nanosci. 2021; 9:1.

http://dx.doi.org/10.7243/2053-0927-9-1 